\title{
Limitações na Prática de Exercício Físico como Fator Desencadeante da Obesidade na População de Baixa Renda
}

\section{Limitations in Exertion as a Starting Factor for Obesity in Low Income Population}

Artigo
Original

Original

Paper

Palavras-chaves:
Exercício Físico
Obesidade

População de Baixa Renda

\section{Resumo}

A obesidade é considerada atualmente uma epidemia mundial que acomete principalmente populações de baixa renda, e uma de suas causas principais é o sedentarismo. Contudo, esses indivíduos possuem limitações para prática de atividades físicas, o que favorece o desenvolvimento da obesidade e dificulta seu tratamento. Este trabalho tem por objetivo compreender as limitações de acesso da população de baixa renda à prática de atividades físicas como parte integrante do tratamento de obesidade. As limitações encontradas foram: nível sócio econômico; falta de informação; necessidade de mães trabalharem fora de casa, tempo em que crianças assistem a televisão, jogam vídeo-game e computador; nível de escolaridade da mãe; urbanização sem planejamento e crescentes taxas de criminalidade. Esses fatores contribuem para uma maior prevalência da obesidade e suas complicações, gerando assim, maior gasto para o governo com a Saúde Pública.

\begin{abstract}
Obesity is nowadays considered a world epidemic which assaults mainly low income populations, and one of its main causes is sedentarism. However, those individuals have limitations for exertion, which favors obesity development and makes its treatment more difficult. This work aims to comprehend limitations of low income population access to physical exercises practice as part of an obesity treatment. The limitations found were: social economic level, lack of information, the necessity of mothers to work outside home, the time children watch television, play video game and computer, mother scholar level, urbanization without planning and the increasing of criminal index. These factors contribute for a larger prevalence of obesity and its complications, requiring, this way, more expenses for government with Public Health.
\end{abstract}

Key words:

Exertion

Obesity

Low Income Population

\section{Introdução}

O excesso de peso é considerado, atualmente, uma ameaça crescente á saúde da população, e entende-se como uma epidemia mundial, sendo uma das causas mais importantes, o sedentarismo.

Segundo a Associação Brasileira De Estudos Sobre Obesidade (ABESO), em
1997 nas regiões nordeste e sudeste do Brasil a prevalência de obesidade e sobrepeso em adultos foi de $38,5 \%$ para homens e $39,0 \%$ para mulheres. Outro achado interessante foi o de que entre 1989 e 1997, na região sudeste, ocorreu uma redução da prevalência de obesidade de $13,2 \%$ para $8,2 \%$ na população 
de maior renda familiar e aumento de $11,6 \%$ para $15,0 \%$ na população de menor renda.

De acordo com Gomes et al (2001) cerca de $60 \%$ dos homens e $78 \%$ das mulheres estudados no Rio de Janeiro não realizava nenhum tipo de atividade física no tempo de lazer.

Em estudo realizado por costa et al (2003), indivíduos que possuem maior renda familiar per capta e maior escolaridade praticaram mais atividades físicas no tempo de lazer, talvez por conhecerem a importância dessas atividades para a saúde.

O nível socioeconômico interfere na disponibilidade de alimentos e no acesso à informação, bem como pode estar associado a determinados padrões de atividade física, constituindo-se, portanto, importante determinante da prevalência da obesidade (Silva et al, 2005).

Contudo, para o tratamento da obesidade, é de fundamental importância o exercício físico e, para população de baixa renda, as limitações são muitas, o que torna necessário uma melhor compreensão dos fatores que combatem a obesidade para que esses indivíduos possam ter uma qualidade de vida melhor.

Devido ao aumento constante de indivíduos obesos no Brasil, este trabalho se justifica pelo fato de que uma das causas para esse problema é a falta de exercícios físicos, o que torna necessário identificar as limitações existentes para prática de tais atividades, proporcionando assim uma melhor qualidade de vida para esses indivíduos.

O objetivo do presente estudo foi o de compreender as limitações de acesso da população de baixa renda à prática de atividades físicas como parte integrante do tratamento de obesidade, entendendo-se a importância do exercício e identificando as limitações encontradas por essa população para tais prática.

\section{Obesidade}

Atualmente, a obesidade é considerada uma epidemia mundial, de acordo com Monteiro \& Conde,(1999) em estudo realizado nas regiões sudeste e nordeste, cerca de $10 \%$ dos adultos são obesos, embora a prevalência se encontre bem distante do um terço encontrados no Estados Unidos, essa porcentagem é preocupante, pois está aumentando nos últimos anos.

A Obesidade é caracterizada pelo acúmulo excessivo de gordura corporal sendo considerada uma ameaça para saúde do indivíduo, acarretando prejuízos como: diabetes mellitus, hipertensão arterial, dislipidemias, doenças cardiovasculares, dificuldades respiratórias, doenças articulares, apnéia do sono e outros problemas respiratórios, contudo, a distribuição corpórea de gordura e as conseqüências para saúde apresentam variações entre os obesos.

Para diagnóstico de obesidade, utiliza-se o índice de massa corpórea (IMC) definido pelo peso em $\mathrm{kg}$ dividido pela altura em metros quadrados, quando, independente do sexo e idade, adultos com IMC igual ou superior á $30 \mathrm{Kg} / \mathrm{m} 2$ devem ser classificados em obesos.(WHO, 1998).

Além do IMC, utiliza-se também, como complemento do diagnóstico, o percentual de gordura corporal e a razão da circunferência da cintura e do quadril, importantes para verificar se o acúmulo de gordura corpórea é na região abdominal ou glúteofemoral e se o indivíduo apresenta riscos de complicações metabólicas.

Vários fatores são importantes na gênese da obesidade, como os genéticos, os fisiológicos e os metabólicos; no entanto, os que poderiam explicar este crescente aumento do número de indivíduos obesos parecem estar mais relacionados às mudanças no estilo de vida e aos hábitos alimentares. (OLIVEIRA \& FISBERG, 2003)

Nesse novo perfil, a urbanização determinou uma mudança nos padrões de comportamento alimentar que, juntamente com a redução da atividade física na população, vem desempenhando importante papel no aumento da prevalência da obesidade no Brasil, proporcionalmente mais elevado nas famílias de baixa renda. (PINEIRO et al, 2000).

Complementando, Barretos et al (2005), relatam que as tendências de aumento da obesidade têm-se mostrado mais acentuadas nos estratos populacionais de menor renda; por outro lado, observa-se a diminuição do excesso de obesidade dos estratos de maior renda.

Para o tratamento da obesidade, é 
imprescindível a combinação de orientações quanto à seleção de alimentos saudáveis, prática de exercícios físicos e modificação do estilo de vida. (MAHAN, 2005).

Sem subestimar os outros fatores que são de extrema importância, no presente trabalho, será abordado somente a prática de exercícios físicos.

Segundo Francischié (2000) é possível reduzir a gordura corporal, sem diminuir o peso quando, por exemplo, ocorre ganho de massa muscular. $\mathrm{O}$ aumento de massa muscular pode ser superior ao peso de gordura reduzido, levando ao aumento no peso corporal total. Assim, a ênfase no tratamento da obesidade deve ser na redução da gordura corporal, já que apenas a perda de gordura promoverá benefícios a saúde.

Além do aumento, a massa corporal magra e, consequentemente, a taxa metabólica basal (TMB) o que contribui para o maior gasto energético e diminuição da massa gorda, o exercício traz outros benefícios como: fortalecimento da integridade cardiovascular, diminuição da resistência à insulina, alívio das tensões e melhora na sensação de bem estar. (MAHAN, 2005).

Contudo, indivíduos de condição sócia econômica mais baixa enfrentam algumas limitações para prática de exercícios físicos, tornando maior a incidência de sedentarismo, contribuindo assim, para maior prevalência de obesidade e, conseqüentemente, contribuindo também para a não economia dos gastos públicos, pois o exercício físico combate diretamente os efeito maléficos á saúde diminuindo assim os custos para saúde pública.(JENOVESI,2004)

\section{Limitações para prática de exercícios físicos}

O nível socioeconômico interfere na disponibilidade de alimentos e no acesso à informação, bem como pode estar associado a determinados padrões de atividade física, constituindo-se, portanto, importante determinante da prevalência da obesidade (Silva et al, 2005), além disso, os alimentos com maior qualidade nutricional, incluindo frutas e verduras, têm custo elevado para as famílias de baixa renda e ao mesmo tempo a indústria alimentícia coloca à disposição vários alimentos com densidade energética aumentada, que promovem saciedade, são mais palatáveis e de baixo custo, o que os torna acessíveis as classe de baixa renda.

Por outro lado, observa-se a dificuldade dessas pessoas de se engajarem em atividades físicas, o que decorre da falta de informações no tocante aos benefícios que o exercício traz à saúde. (SILVA et al, 2005).

Em um estudo, realizado na cidade de Montes Claros em Minas Gerais por (Pereira \& Barros, 2004), mostrou que a maioria dos escolares, independentemente da classe social, assistem a televisão em aproximadamente $30 \mathrm{~h}$ /semana, ou seja, de 3 h á 4 h e $30 \mathrm{~min} /$ dia. É importante lembrar que, além da televisão, existem outras atividades como videogame e computadores que ocupam um tempo disponível que poderia ser empregado na participação em atividades físicas.

Em controvérsia, Hallal et al, (2006) verificaram que quanto maior o tempo de uso de computador e videogame, maior o percentual de atividade física no lazer. Ao contrário, reforçando o estudo citado acima, quanto menor o tempo assistindo à televisão, maior a prevalência de atividade física no lazer.

Uma possível explicação para o efeito do videogame é que grande parte dos jogos é de lutas e esportes, o que poderia servir como estímulo à prática de atividade física entre os jovens.

É bem provável que os hábitos de atividade física adquiridos na infância possam influenciar no nível de atividade física na idade adulta, já que a prática se torna um hábito, tendo um efeito positivo na qualidade de vida, prevenindo doenças dentre elas a obesidade e suas complicações.

Citando novamente Hallal et al (2006), os autores verificaram que as mães de adolescentes de baixa renda apresentaram, em maior proporção obesidade e menos prática de atividades físicas no momento de lazer, mostrando um fator de risco para o sedentarismo em seus filhos, que praticavam menos atividades físicas quando comparados com adolescentes de classe mais alta.

Esse achado demonstra que os hábitos são formados de acordo com o meio onde a criança está inserida, que pode no caso das de baixa renda favorecer futuramente $o$ aparecimento de complicações para a saúde, 
já que, uma criança obesa tem mais chances de se tornar um adulto obeso.

Outro fator importante é o grau de escolaridade da mãe, segundo estudo realizado na região sul do Brasil por Oehlschlaeger et al, (2004), adolescentes cujas mães apresentam níveis de escolaridade menor de quatro anos, possuem duas vezes mais chances de desenvolverem comportamento sedentário quando comparados com filhos de mães que possuem nove ou mais anos de escolaridade.

Em estudo realizado por Bracco et al, (2006), em escolas públicas da cidade de São Paulo, o risco para o sedentarismo é maior na população de baixa renda e em casas onde as mães trabalham fora.

Uma explicação para tal fato poderia ser que a condição econômica mais vulnerável, na maioria das vezes, é causada pela baixa escolaridade, tornando necessário que mães trabalhem fora de casa. Uma situação bastante comum nesses lares é que os filhos ficam em casa, com os mais velhos tomando conta dos menores. Assim, existem menos oportunidades para a prática de atividade física fora da escola entre essas crianças.

Outro achado importante por Bracco et al, (2006), foi que vizinhanças menos favorecidas por serviços à comunidade podem ser um fator para a promoção de comportamento sedentário entre crianças, além disso, a urbanização sem planejamento, no que se refere aos espaços públicos, e as crescentes taxas de criminalidade em centros urbanos são grandes barreiras para a prática de atividade física entre crianças, promovendo, como conseqüência, comportamentos inativos relacionados com opções de lazer restritas atividades com baixo gasto energético.

Pereira \& Barros et al (2004) concluíram que as crianças de nível sócio-econômico mais baixo foram significativamente mais ativas do que os de alto nível sócio-econômico. As diferenças podem ser explicadas pelo fato de que as crianças de nível socioeconômico mais baixo apresentam pobres condições de vida a qual as força a praticar mais atividades de manutenção da casa e caminhar para a escola, uma vez que os que tinham o nível socioeconômico mais elevado eram transportados de carros e tinham mais tempo para assistir à televisão.

Entende-se que indivíduos menos favorecidos possuem algumas limitações para prática de exercícios físicos no lazer, mas suas atividades diárias devido às necessidades são mais ativas, quando comparados com pessoas de uma melhor renda.

\section{Considerações finais}

Pode-se concluir que as limitações existentes para prática de atividades físicas em indivíduos de baixa renda vão desde a falta de informação sobre a importância dessas atividades para saúde até a deficiência de planejamento urbano para a prática, ou seja, falta de praças e calçadas, por exemplo.

Deve-se salientar ainda, que a falta de informação é devido a baixa escolaridade, que por sua vez é causada pela necessidade de se trabalhar fora de casa, o que torna mães obrigadas a deixarem seus filhos maiores tomando conta dos menores o que por sua vez os impedem de praticar atividades físicas, aumentando assim, as chances de obesidade e complicações futuras.

Já que essas crianças passam o maior tempo em casa, consequentemente, o tempo que essas assistem à televisão também é maior, e o hábito para tal começa então a ser formado desde já, contribuindo para uma vida adulta sedentária com doenças crônicas não transmissíveis, gerando maior custo para saúde pública.

Outro problema é a oferta de alimentos calóricos ricos em açúcar e gordura, de baixo custo, que essa população tem acesso mais facilitado, o que acaba sendo um fator contribuinte para obesidade.

Percebe-se então, que a causa e tratamento da obesidade são complexos, e que medidas curativas e preventivas devem ser tomadas urgentemente, proporcionando uma melhor qualidade de vida aos indivíduos de baixa renda.

\section{Referências}

BARRETO,S.M et al;Análise da Estratégia Global para Alimentação, Atividade Física e Saúde.Epidemiologia e Serviços de Saúde. Vol.14.n1.p.41 - 68 .

BRACCO,M.M.et al.Modelo hierárquico multivariado da inatividade física em crianças 
de escolas públicas. São Paulo. Jornal de Pediatria . Vol. 82, Nº4. 2006.

COSTA,R.S et al;Associação entre fatores sócio-demográficos e prática de atividade física de lazer no Estudo Pró-Saúde.Rio de janeiro. Caderno de Saúde Pública. Vol.19. n4.p1095-1105. jul/ago, 2003.

FRANCISC HI,R.P.P et al; Obesidade: Atualização Sobre Sua Etiologia, Morbidade e Tratamento. Revista De Nutrição De Campinas. Campinas.Vol.13.n1.p.17-28,jan/ abr.2000

GOMES, V. B.; SIQUEIRA, K. S; SICHIERI, R.Atividade física em uma amostra probabilística do Município do Rio de Janeiro.Cadernos de Saúde Pública..Vol.17. n4.p.969-976.jul/ago.2001

HALLAL,P.Cetal.Prevalênciadesedentarismo e fatores associados em adolescentes de 10-12 anos de idade. Caderno de Saúde Pública. Rio de Janeiro. Vol.22.n.6. p.1277-1287. jun, 2006.

JENOVESI, J. F et al. Evolução no nível de atividade física de escolares observados pelo período de 1 ano. . Revista Brasileira Ci e Movimento.Vol.12.n1.p.19 - 24. 2004

MAHAN,L KATHLEEN. ESCOTT-STUMP, SYLVIA. KRAUSE. Alimentos, nutrição e dietoterapia. Vol11. São Paulo, Brasil: Roca, 2005.p1242.

MONTEIRO, C. A.;CONDE, W. L. A tendência secular da obesidade segundo estratos sociais: Nordeste e Sudeste do Brasil, 1975-1989-1995. Arquivos Brasileiros de Endocrinologia e Metabologia .Vol.43. n3.p.186 -194.jun.1999

OEHLSCHLAEGER,M.H et al. Prevalência e fatores associados ao sedentarismo em adolescentes de área urbana. Revista de Saúde Pública. Vol.38. n.2 p.157-63. 2004

OLIVEIRA.C.L;FISBERG.M.Obesidade na Infância e Adolescência - uma verdadeira Epidemiologia. Arquivo Brasileiro Endocrinologia e Metabologia. Vol.47. n2,abr. 2003
PEREIRA,R.R;BARROS,J.F.Estilo de vida dos escolares de Montes Claros, MG.Revista

Digital. Buenos Aires. Vol.10. $\mathrm{N}^{\circ}$ 75.ago. 2004

PINHEIRO,A.R.O;

FREITAS,S.F.T; CORSO,A.F.T. Uma abordagem epidemiológica da obesidade. Revista de Nutrição. Campinas. Vol17. n 4 p.523-533, out./dez.. 2004

SILVA,G.A.P;BALABAN,G;MOTTA,M.E.F. A. Prevalência de sobrepeso e obesidade em crianças e adolescentes de diferentes condições socioeconômicas.Revista Brasileira De Saúde Materno Infantil. Recife.Vol.5. n1.p.53-59, jan. / mar. 2005.

World Health Organization. Obesity: preventing and managing the global endemic. Report of a WHO consultation. Geneva: WHO; 2000. WHO Technical Report Series, 894.

Endereço para Correspondência:

Nívea Veiga Almeida

Cursos de Nutrição

nivea.v.a@hotmail.com

Centro Universitário de Volta Redonda

Campus Três Poços

Av. Paulo Erlei Alves Abrantes, $n^{\circ} 1325$

Três Poços - Volta Redonda / RJ

CEP: $27240-560$

Informações bibliográficas:

Conforme a NBR 6023:2002 da Associação Brasileira de Normas Técnicas (ABNT), este texto científico publicado em periódico eletrônico deve ser citado da seguinte forma: ALMEIDA, N. V.. Limitações na Prática de Exercício Físico como Fator Desencadeante da Obesidade na População de Baixa Renda, Volta Redonda, ano III, n. 8, dezembro. 2008. Disponível em: <http://www.unifoa.edu.br/pesquisa/caderno/edição/08/77.pdf> 